
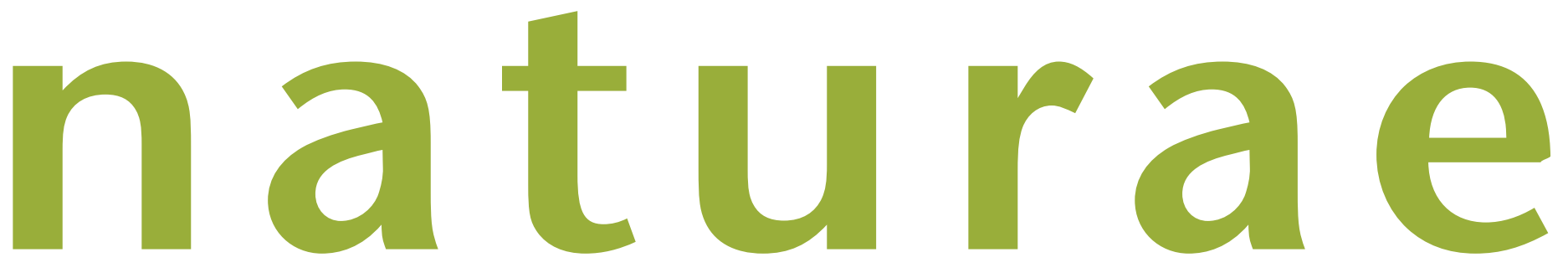

$2018 \cdot 4$

Distribution statistique de la taille imaginale de Pterostichus madidus (Fabricius, 1775) dans les plaines de l'est de la France: de la nécessité de rechercher ses composantes géographiques et historiques

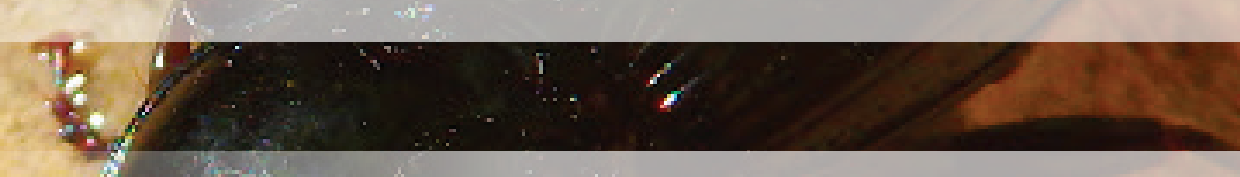

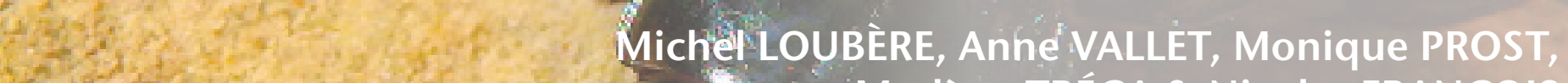
14 20. Marlène TRÉCA \& Nicolas FRANÇO 36 
DiRECTEUR DE LA PUbliCATION: Bruno David,

Président du Muséum national d'Histoire naturelle

RÉDACTEUR EN CHEF / EDITOR-IN-CHIEF: Jean-Philippe Siblet

ASSISTANTE DE RÉDACTION / ASSISTANT EDITOR: Sarah Figuet (naturae@mnhn.fr)

Mise EN PAGE / PAGE LAYOUT: Sarah Figuet

COMITÉ SCIENTIFIQUE / SCIENTIFIC BOARD:

Luc Abbadie (UPMC, Paris)

Luc Barbier (Parc naturel régional des caps et marais d'Opale, Colembert)

Aurélien Besnard (CEFE, Montpellier)

Vincent Boullet (Expert indépendant flore/végétation, Frugières-le-Pin)

Hervé Brustel (École d'ingénieurs de Purpan, Toulouse)

Audrey Coreau (AgroParis Tech, Paris)

Thierry Dutoit (UMR CNRS IMBE, Avignon)

Éric Feunteun (MNHN, Dinard)

Grégoire Gautier (Parc national des Cévennes, Florac)

Olivier Gilg (Réserves naturelles de France, Dijon)

Frédéric Gosselin (Irstea, Nogent sur Vernisson)

Patrick Haffner (UMS PatriNat, Paris)

Frédéric Hendoux (MNHN, Paris)

Xavier Houard (OPIE, Guyancourt)

Isabelle Leviol (MNHN, Paris)

Francis Meunier (Conservatoire d'espaces naturels - Picardie, Amiens)

Serge Muller (MNHN, Paris)

Francis Olivereau (DREAL Centre, Orléans)

Laurent Poncet (UMS PatriNat, Paris)

Nicolas Poulet (ONEMA, Toulouse)

Jean-Philippe Siblet (UMS PatriNat, Paris)

Laurent Tillon (ONF, Paris)

Julien Touroult (UMS PatriNat, Paris)

COUVERTURE / COVER:

Pterostichus madidus (Fabricius, 1775) dans la réserve naturelle régionale et Forêt d'exception de Val-Suzon. Crédit photo: M. Loubère.

Naturae est une revue en flux continu publiée par les Publications scientifiques du Muséum, Paris / Naturae is a fast track journal published by the Museum Science Press, Paris

Les Publications scientifiques du Muséum publient aussi / The Museum Science Press also publish:

Adansonia, Anthropozoologica, European Journal of Taxonomy, Geodiversitas, Zoosystema.

Diffusion - Publications scientifiques Muséum national d'Histoire naturelle

CP 41 - 57 rue Cuvier F-75231 Paris cedex 05 (France)

Tél. : 33 (0)1 40794805 / Fax: 33 (0)1 40793840

diff.pub@mnhn.fr/http://sciencepress.mnhn.fr

(c) Publications scientifiques du Muséum national d'Histoire naturelle, Paris, 2018

ISSN (imprimé / print): 1280-9551/ ISSN (électronique / electronic): 1638-9387

PHOTOCOPIES :

Les Publications scientifiques du Muséum adhèrent au Centre Français d'Exploitation du Droit de Copie (CFC), 20 rue des Grands Augustins, 75006 Paris. Le CFC est membre de l'International Federation of Reproduction Rights Organisations (IFRRO). Aux Etats-Unis d'Amérique, contacter le Copyright Clearance Center, 27 Congress Street, Salem, Massachusetts 01970.
PHOTOCOPIES:

The Publications scientifiques du Muséum adhere to the Centre Français d'Exploitation du Droit de Copie (CFC), 20 rue des Grands Augustins, 75006 Paris. The CFC is a member of International Federation of Reproduction Rights Organisations (IFRRO). In USA, contact the Copyright Clearance Center, 27 Congress Street, Salem, Massachusetts 01970. 


\title{
Distribution statistique de la taille imaginale de Pterostichus madidus (Fabricius, 1775) dans les plaines de l'est de la France: de la nécessité de rechercher ses composantes géographiques et historiques
}

\author{
Michel LOUBÈRE \\ Société entomologique de Dijon, Laboratoire d'entomologie, \\ Muséum-Jardin des Sciences de Dijon, 14 rue Jéhan de Marville, F-21000 Dijon (France) \\ et Société Lorraine d'entomologie, Muséum-Aquarium de Nancy, \\ 34 rue Sainte-Catherine, F-54000 Nancy (France) \\ malachius@orange.fr
}

Anne VALLET

Société Lorraine d'entomologie, Muséum-Aquarium de Nancy, 34 rue Sainte-Catherine, F-54000 Nancy (France) entomo.logic54@gmail.com

Monique PROST

Société entomologique de Dijon, Laboratoire d'entomologie, Muséum-Jardin des Sciences de Dijon, 14 rue Jéhan de Marville, F-21000 Dijon (France) monique.prost043@orange.fr

Marlène TRÉCA

Réserve naturelle régionale et Forêt d'exception de Val-Suzon, Agence ONF Bourgogne Est, 11 rue René Char, F-21000 Dijon (France) marlene.treca@onf.fr

Nicolas FRANÇOIS

Réserve naturelle nationale Combe Lavaux-Jean Roland, 2 rue des Halles, F-21220 Gevrey-Chambertin (France) nicolas.francois@espaces-naturels.fr

Soumis le 6 mars 2018 | Accepté le 25 mai 2018 | Publié le 3 octobre 2018

\begin{abstract}
Loubère M., Vallet A., Prost M., Tréca M. \& François N. 2018. - Distribution statistique de la taille imaginale de Pterostichus madidus (Fabricius, 1775) dans les plaines de l'est de la France: de la nécessité de rechercher ses composantes géographiques et historiques. Naturae 2018 (4): 43-55. http://revue-naturae.fr/2018/4

\section{RÉSUMÉ}

La taille imaginale de Pterostichus madidus (Fabricius, 1775) est définie comme la distance entre l'apex des mandibules au repos et l'apex des élytres. Sa variabilité est explorée dans les dimensions latitudinales, altitudinales et temporelles de sa niche écologique, à l'aide d'un jeu de données constitué des mesures de 658 spécimens collectés de 2007 à 2016 dans plusieurs espaces protégés et milieux ordinaires de Côte-d'Or, Meurthe-et-Moselle et Moselle. Les femelles se montrent toujours les plus réactives. Un gradient de variation latitudinale de type Bergmann inversé leur est trouvé. Au sud, elles sont plus grandes de $0,7 \mathrm{~mm}$ en moyenne. Chez les mâles se constatent une hétérogénéité de la variance et une variabilité stationnelle qui rendent leur gradient latitudinal moins perceptible. Mais il n'y a pas de variation significative du dimorphisme sexuel avec la latitude. Les animaux récoltés sur la période 2012-2016 en Côte-d'Or sont comparés à un échantillon collecté dans les
\end{abstract}




\section{MOTS CLÉS \\ Coleoptera, \\ Carabidae, \\ latitude, \\ changement climatique.}

mêmes secteurs de 1978 à 1998. Les femelles récentes furent trouvées plus grandes de 0,7 mm, ce qui fait un premier cas de variation inter-générationnelle de la taille imaginale pour un coléoptère terrestre dans son milieu naturel, ici à l'échelle d'un département. L'éventualité qu'il s'agisse d'une réponse au changement climatique est considérée, en regard de ce qu'il reste à apprendre sur l'écophysiologie larvaire de cette espèce en milieu naturel et des réactions prévues aux poïkilothermes terrestres dans ce contexte.

\begin{abstract}
Statistical distribution of Pterostichus madidus (Fabricius, 1775) body size in eastern France lowlands: in need of a search for its geographical and historical components

Pterostichus madidus (Fabricius, 1775) imaginal body size is defined as the distance between elytron and mandible tips, when mandibles lay in their resting position. Its statistical distribution was estimated from 658 specimens, that had been pitfall-trapped in the 2007-2016 year span along a northward transect across the French lowlands of Côte-d'Or, Meurthe-et-Moselle and Moselle. The sampling targeted exploited, protected and unmanaged woodlands. It involved one national and two regional nature reserves. No response to the deliberately restricted elevational range was detected. Females displayed the more obvious response to the latitudinal shift, which took the form of a steep inversed Bergmann's cline. The ones, that originated from the transect southernmost end were found to be 0.7 millimeters longer on average. Though males variance turned out to be heterogeneous and their latitudinal cline to be made looser by some important local variation, no significant southward increase of sexual dimorphism could be demonstrated. Specimens pooled from our 2012-2016 collections in Côte-d'Or were compared to an additional museum sample, that gathered beetles obtained from the same or neighboring locations in 1978-1998. It was observed, that the 2012-2016 females had lengthened by an average amount of $0.7 \mathrm{~mm}$, thus yielding a first coleopteran case of a generational shift for a terrestrial species observed within its natural range and under natural conditions. Given the phenomenon spatial scale, the likelihood of it being a response to climate change is discussed considering knowledge gaps about larval trophic network and theoretical models of ectotherm organisms reaction to temperature increase.
\end{abstract}

\section{INTRODUCTION}

La biomasse de la litière détermine pour une large part celle de ses décomposeurs, desquels se nourrissent de nombreuses espèces de carabiques qui en sont des régulateurs. Cette relation trophique explique que la biomasse des carabiques puisse être considérée comme un indicateur de l'état de la succession forestière (Kotze et al. 2011), ce qui a pu être confirmé sur de vastes échantillons grâce au gain de temps expérimental rendu possible par l'existence d'une relation allométrique (voir Annexe 1) entre la biomasse et la taille de l'individu (Schreiner \& Schwerk 2012). Le profit à retirer de l'utilisation de cet indicateur est conditionné à la précision de l'estimation de la taille. On peut se retrouver obligé de s'en remettre aux estimateurs publiés, par manque de temps pour effectuer cette mesure ou confronté à des espèces qui viennent par exemplaires isolés, rendant impossible le calcul d'une moyenne. La littérature fournit habituellement les tailles inférieures et supérieures, avec une précision millimétrique, sans donner de détails sur la distribution statistique ou la manière dont le dimorphisme sexuel peut l'affecter. D'une référence à l'autre s'observent des fluctuations parfois importantes et rarement discutées. Jeannel (1942) partagea nos interrogations. En le suivant dans une comparaison des références à Pterostichus madidus (Fabricius, 1775) du nord au sud de l'Europe, on se forme la même impression de schéma, dont lui-même rendit compte dans une rédaction variétiste, qui reflétait l'approche développée de son temps. Il y a presque une unanimité au nord au sujet de la taille des plus petits spécimens, qui paraissent former une classe naine de 13 millimètres (Lindroth 1974). Du Royaume-Uni à l'Espagne, les estimateurs de la plus grande taille dessinent pratiquement un gradient géographique nordsud. En France, pays en position médiane dont le gradient latitudinal empiète largement sur celui de l'Allemagne, se rencontrerait une variabilité qui engloberait toutes les autres.

Les modèles embryologiques font de la variabilité de la taille adulte une expression des fluctuations du contrôle du taux de croissance larvaire, ainsi que du moment de la survenue du signal hormonal interrompant la croissance du dernier stade larvaire. Celui-ci est le résultat d'une chaîne d'événements physiologiques. Elle démarre quand la biomasse critique est atteinte, ce qui explique la relation entre taille et biomasse. Mais la complexité du système le laisse ouvert à l'influence par d'autres paramètres environnementaux. Par exemple, une température élevée favorise les grosses variétés de la coccinelle Harmonia axyridis (Pallas, 1773) (Soares et al. 2003) ou conduit au contraire à une réduction de la taille imaginale chez notre carabique Notiophilus biguttatus (Fabricius, 1779) (Ernsting \& Isaaks 1987, 1997; Ernsting et al. 1992). Dans la famille des Carabidae Latreille, 1802, il semble que chaque espèce ou chaque sexe réagisse à sa manière aux gradients édaphiques (voir Annexe 1), par l'indifférence ou par une variation de la taille imaginale dans le sens du gradient ou contre lui. 
D'un autre point de vue, cette impression première de gradient latitudinal pourrait être contredite, considérant que Jeannel (1942) a pu faire des confusions au sud du Massif central avec le grand Pterostichus validus (Dejean, 1828) (Coulon et al. 2011). Aussi, un niveau de précision millimétrique implique des erreurs d'arrondi.

Par la définition allométrique (voir Annexe 1) de la relation, l'effet de toute variation de taille sur l'estimation de la biomasse est multiplicatif. D'après Jeannel (1942), les P. madidus français seraient $20 \%$ plus grands et donc $80 \%$ plus gros que leurs cousins germaniques et britanniques. Les données sur l'activité-densité forestière de cette espèce (Toïgo et al. 2013) la placent en position de codominance avec Abax parallelepipedus (Piller \& Mitterpacher, 1783). Les conséquences d'une telle approximation pour les estimateurs des communautés peuvent alors se révéler assez considérables. Faute d'idée des caractéristiques de la distribution statistique, il ne saurait être question d'éluder ce problème en se réfugiant derrière une hypothétique rareté de ces géants. Artefact ou gradient latitudinal, il va nous falloir trancher. Aussi, cet article se propose-t-il de mettre à l'épreuve l'hypothèse nulle d'une variation strictement fortuite de la taille imaginale au travers d'une emprise géographique, certes réduite à l'échelle du continent, mais suffisamment élargie pour que le gradient latitudinal commençât d'y être sensible.

\section{MATÉRIEL ET MÉTHODES}

\section{DÉFINITION ET MESURE DE LA TAILLE}

Les exemplaires modernes furent séchés à l'air libre sur buvard, sous lampe de 40 watts. Un sous-échantillon de huit femelles et cinq mâles a été suivi longitudinalement pendant 350 heures, afin de vérifier que les mesures ont été faites au-delà de la durée de séchage des articulations céphalo-thoraciques et pro-mésothoraciques. En vue dorsale, afin de s'affranchir de la souplesse abdominale des femelles gravides (Lpy dans la Fig. 1), nous avons relevé l'équivalent de l'estimateur SBL de Babin-Fenske et al. (2008), qui mesure la distance des mandibules à l'apex de l'élytre (LAE dans la Fig. 1), dont la valeur a été calculée au microscope électronique (grossissement de 20, précision de 40 microns) ainsi qu'au pied à coulisse pour contrôle. Les mesures sont exprimées avec une précision du décimillimètre. Les échantillons historiques ont été mesurés à l'oculaire micrométrique calibré contre un graticule (voir Annexe 1). Les exemplaires muséaux, desquels les mandibules avaient été ouvertes à l'occasion de précédents travaux d'anatomie mandibulaire, furent écartés.

\section{LES ÉCHANTILLONS}

\section{Données contemporaines}

Afin de restreindre le risque d'interférences que pourraient induire une variation du cycle reproducteur avec l'altitude (Butterfield 1996) ou de trop grandes différences de substratum (voir Annexe 1) (Tyler 2008), nous nous sommes cantonnés aux plaines de Meurthe-et-Moselle, Moselle et Côte-d'Or (Fig. 2). Mais nous préférerons un découpage

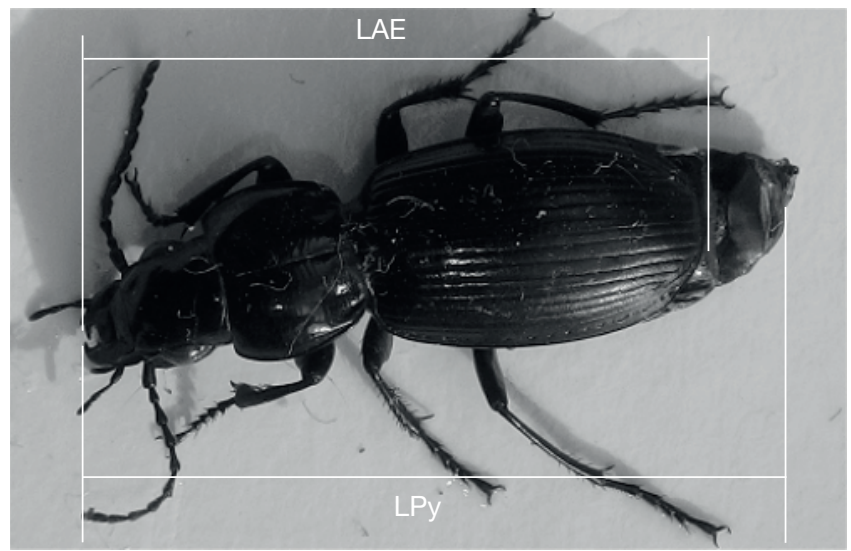

FIG. 1. - Les possibilités de mesurer la taille imaginale phénotypique et les différences de valeurs qu'elles impliquent illustrées par le cas d'une femelle Pterostichus madidus (Fabricius, 1775) du 22 mai 2008, originaire de la Forêt domaniale de Haye. Les mesures de longueur possibles partent de l'apex mandibulaire et diffèrent par leur point d'arrêt: LAE (mesure jusque l'apex de l'élytre) et LPy (mesure jusqu'au pygidium).

territorial plus signifiant pour cette espèce forestière en plaine, les Régions forestières nationales (RFN), ici désignées par les codes numériques de l'Inventaire forestier national: 216 (Montagne Bourguignonne), 544 (la Haye), 572 (Plateau Lorrain), 702 (Plateau Haut-Saônois) et 712 (la Côte). Elles sont englobées dans deux sylvo-éco-régions (Cavaignac 2009): C30 pour le Plateau Lorrain («plaines et dépressions argileuses du nord-est de la France») et C20 pour toutes celles prises en compte dans les analyses des gradients latitudinal et historique ("plateaux calcaires du nord-est de la France»).

La Haye a fourni une remarquable profusion de spécimens (Fig. 3). Certes, il y règne des conditions subcontinentales bien plus optimales pour $P$. madidus. Mais il y a aussi fort à suspecter que nos boisements, épargnés en 1999 par Lothar (Fig. 4A), jouaient un rôle de refuge pour la faune forestière des alentours sinistrés.

Par le jeu de la topographie et de l'assise de la Réserve naturelle régionale \& Forêt d'exception de Val-Suzon à cheval sur les RFN 216 et 712, les stations de ces deux RFN se trouvent pour une partie d'entre elles à des latitudes comparables (Fig. 2). Ce sont le relief et le substrat qui se combinent alors pour y engendrer un gradient de thermophilie assez fort de l'une à l'autre (Fig. 4B et F). Abritée des vents d'ouest et située au-dessus des brumes du Val de Saône (Inventaire forestier national 2008), la côte (RFN 712) est encore asséchée par son substrat karstique qui engloutit promptement des précipitations déjà diminuées de $200 \mathrm{~mm}$ en moyenne annuelle. La topographie donne aux calcaires bajociens et bathoniens (voir Annexe 1), qui affleurent un peu partout (Fig. 4D-F), toute latitude pour exprimer leur potentiel d'échauffement. Celui-ci produit l'exubérance du buis (Buxus sempervirens L.) qui envahit les landes desséchées et les taillis combustibles (Fig. 4D et E). Ce milieu plutôt hostile produisit logiquement le moins de spécimens (Fig. 3). 


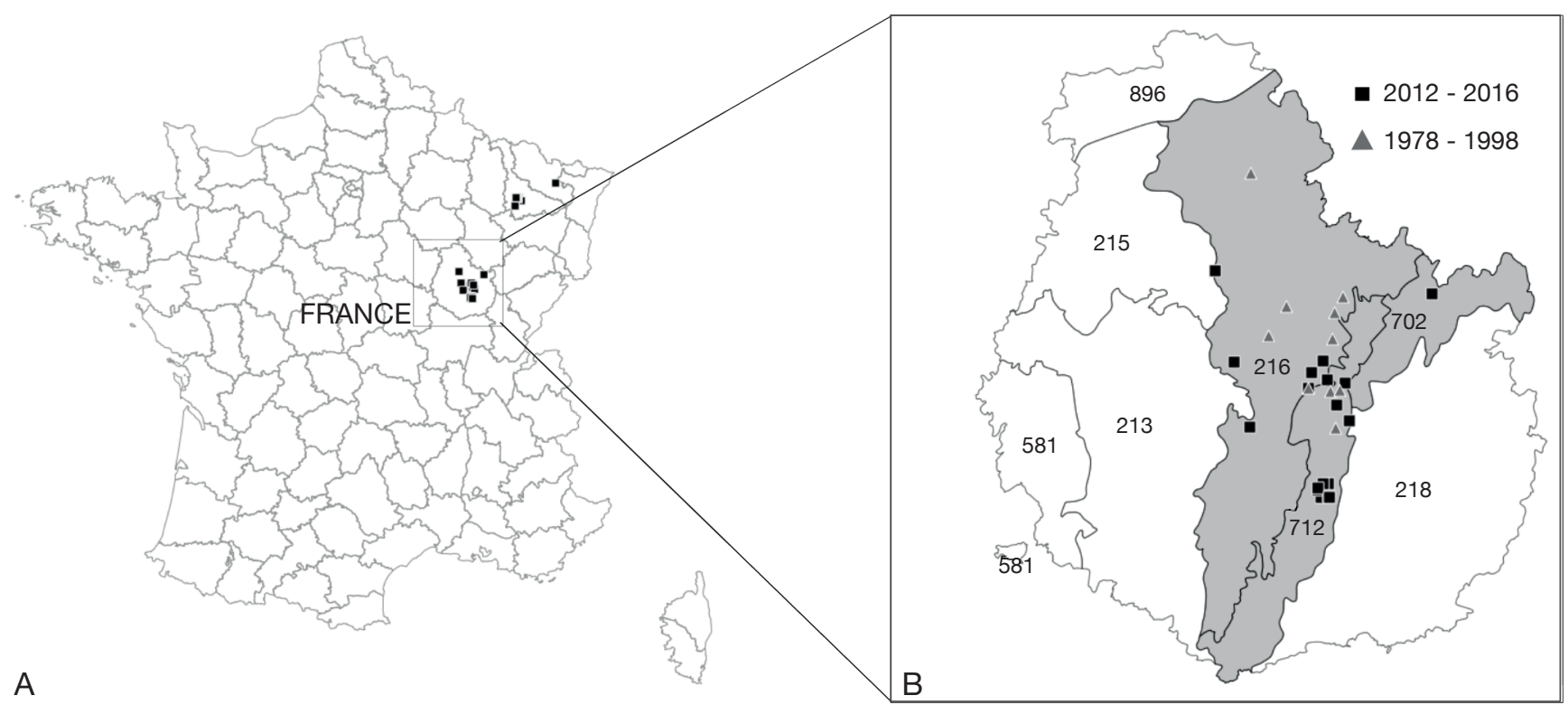

FIG. 2. - Situation des prélèvements. A, transect nord-sud au travers de la Moselle, la Meurthe-et-Moselle et la Côte-d'Or. Le rectangle indique l'emprise du zoom; B, grossissement de la carte de Côte-d'Or afin d'indiquer l'emplacement des relevés de l'échantillon 2012-2016 et de l'échantillon 1978-1998. Les divisions internes sont les limites des régions forestières nationales, qui sont identifiées par leur code numérique IFN. Celles concernées par la présente étude apparaissent en gris: 216 (Montagne Bourguignonne), 702 (Plateau Haut-Saônois), 712 (Côte et arrière-côte de Bourgogne).

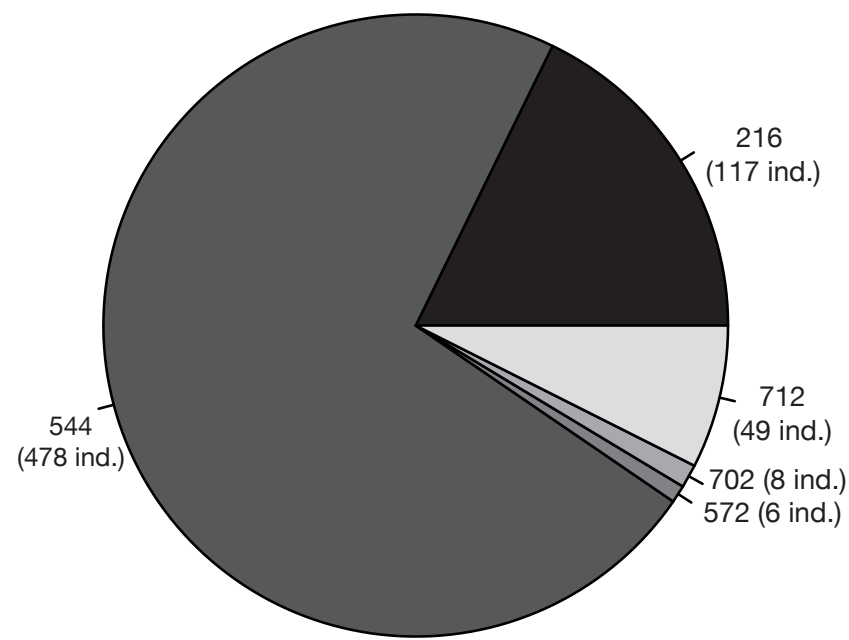

FIG. 3. - Ventilation des données par région forestière nationale: Montagne Bourguignonne (216), Plateau de Haye (544), Plateau Lorrain (572), Plateau Haut-Saônois (702), Côte et arrière-côte de Bourgogne (712).

Sur 36 stations, 29 ont été équipées chacune de trois pièges barbers (un exemplaire dans la Fig. 4D) opérés pendant au moins deux ans, remplis d'un mélange de saumure additionnée de teepol comme produit mouillant. Les sept dernières avaient un seul piège, car elles participaient à un travail d'atlas, où il n'y avait pas l'objectif de comparer les mesures d'activité-densité.

À la saison des amours, les mâles tendent à circuler davantage que les femelles, c'est pourquoi ils sont en léger excès dans les données (363 mâles pour 295 femelles). Les périodes d'échantillonnage balaient l'année d'avril à septembre. Une météorologie inhabituellement clémente rendit parfois possible de poursuivre jusqu'en novembre.

\section{Données historiques}

Le peu de données sur les variations inter-annuelles de la taille imaginale des carabiques les font paraître insignifiantes (Gouws 2007). Néanmoins et dans le but de nous ôter tout doute quant à l'origine réelle des différences éventuellement exprimées par les individus au nord (période 2007-2009) et au sud du transect (période 2012-2016), nous avons mesuré un lot additionnel de 27 femelles et 17 mâles, récoltés de 1978 à 1998 en Côte-d'Or (par Mrs Perrot et Darge, ainsi que Mme Prost), fournis par le Muséum-Jardin des Sciences de Dijon, que nous nous proposons de comparer à ceux des nôtres qui proviennent des mêmes RFN (Fig. 2), dont certains des mêmes communes, afin de vérifier la stabilité de LAE sur un intervalle de temps qui encadre l'époque de nos mesures dans la Haye.

\section{ANALYSE DES DONNÉES Variables indépendantes}

Il y a trois variables indépendantes, qui font l'objet de tests, qui sont notées: RFN, ALT et Période.

Nous avons deux niveaux de hiérarchisation géographique: station et RFN. La grande disparité d'effectifs entre les stations, ainsi que la nécessité de maintenir un équilibre dans la représentation des mâles et des femelles imposèrent le regroupement des stations par RFN et seules les RFN avec un nombre suffisant de mâles et de femelles furent conservées, soit 216, 544 et 712 (Fig. 2 et 3).

Quoique nous ayons délibérément réduit l'amplitude des variations altimétriques, il arrive parfois qu' elles correspondent à des transitions écologiques abruptes, dans les zones de cuesta (voir Annexe 1) de la Côte et de la Haye principalement. L'altitude ne peut être testée que du côté de la Côte-d'Or, où 

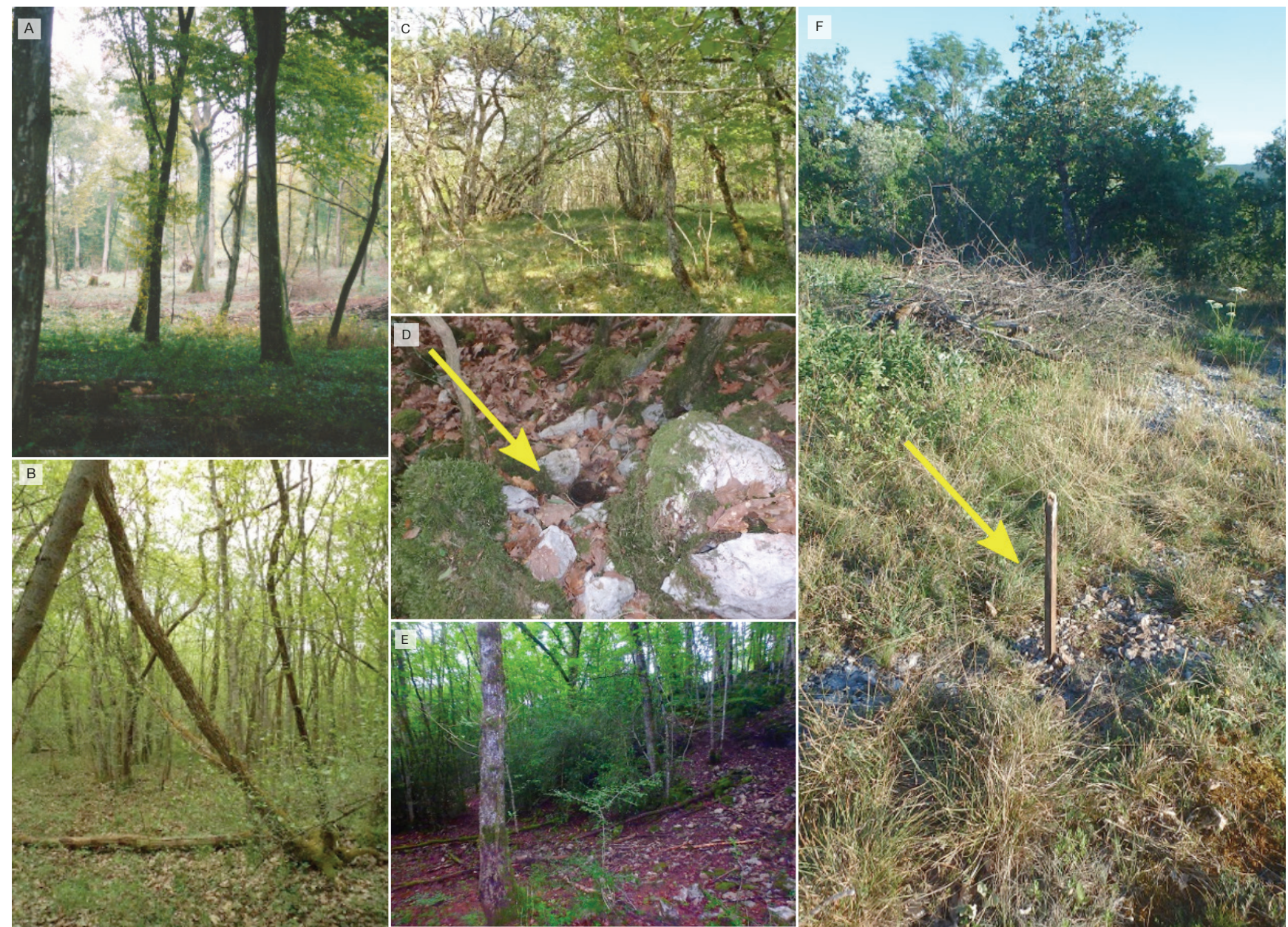

FIG. 4. - Aperçu des conditions édaphiques (voir Annexe 1) de l'échantillonnage 2007-2016. A, cliché pris dans le Bois de Gondreville (RFN 544) afin de montrer la difficulté de notre quête d'un boisement survivant d'une étendue suffisante pour être exempt d'effet de lisière, de manière à avoir conservé le micro-climat forestier préexistant à Lothar; B, îlot de sénescence Goa dans la RNR \& Forêt d'exception de Val-Suzon, Montagne Bourguignonne (RFN 216); C, station RVS2 de la Montagne Bourguignonne (RFN 216) dans la RNR \& Forêt d'exception de Val-Suzon, dont les données ont été utilisées pour le calcul des indices de dimorphisme sexuel; D, station 84 de la Réserve naturelle nationale Combe Lavaux-Jean Roland dans la Côte (RFN 712) avec affleurements de blocs de calcaire (noter le piège barber fléché au centre des blocs) dans un sous-bois de buis, où l'activité-densité de Pterostichus madidus (Fabricius, 1775) a été trouvée résiduelle à l'instar de celle de tous les autres carabiques forestiers; E, îlot de senescence Ragot de la RNR \& Forêt d'exception de Val-Suzon dans la Côte (RFN 712) avec sous-bois de buis et délitement calcaire en laves, site d'origine des données de Mr Darge; $\mathbf{F}$, lande et forêt sur cailloutis calcaires (RFN 712) dans la RNR \& Forêt d'exception de Val-Suzon (noter le jalon fléché qui repère le centre de la station). Crédits photos : M. Loubère.

les sites s'étagent de 200 à 500 mètres. Nous les avons répartis dans trois classes (variable ALT) numérotées depuis 2 (points d'altitude comprise entre 200 et 299 mètres) jusque 4 (points d'altitude supérieure à 400 mètres).

La répartition des données historiques n'est pas homogène dans le temps. Elles durent donc être regroupées, de sorte que la variable Période n'ait que deux modalités: contemporaine et ancienne.

\section{Ajustement et correction des déséquilibres}

La possibilité d'utiliser la distribution normale comme base de l'analyse a été évaluée par inspection visuelle de la distribution expérimentale et calcul de ses moments de symétrie et d'aplatissement (David et al. 1997). Les modèles ont été ajustés à la variable dépendante centrée et réduite:

$$
\mathrm{lae}_{\mathrm{i}}=\left(\mathrm{LAE}_{\mathrm{i}}-\mu\right) / \sigma
$$

où i est l'individu, $\mu$ et $\sigma$ sont la moyenne et l'écart-type expérimentaux, calculés sur toutes les $\mathrm{RFN}$. $\operatorname{lae}_{\mathrm{i}}<0$ identifie un sujet plus petit que la moyenne et inversement s'il est plus grand $\left(\operatorname{lae}_{\mathrm{i}}>0\right)$. Un écart de 1 unité de lae signifie que l'individu a réalisé une valeur de LAE éloignée de la moyenne de l'espèce de 1 écart-type.

S'étant déjà vu chez les Carabiques, que les morphologies mâles et femelles puissent répondre différemment à un gradient édaphique (voir Annexe 1), nous avons fait l'hypothèse d'un modèle emboîté à deux paramètres pour chacune des trois variables indépendantes:

$$
\text { lae }_{\mathrm{ijk}}=\mu+\alpha_{\mathrm{k}}+\beta_{\mathrm{j} \mid \mathrm{k}}+\varepsilon_{\mathrm{ijk}}, \varepsilon_{\mathrm{ijk}}-\mathrm{N}\left(0, \sigma_{\varepsilon}^{2}\right)(1)
$$

avec pour l'individu i, la modalité j de la variable indépendante (qui peut être RFN, ALT ou Période) et le sexe k: $\alpha_{\mathrm{k}}$ la variation attribuable au sexe $\mathrm{k}, \beta_{\mathrm{j} \mid \mathrm{k}}$ le paramètre pour la j-ième modalité de la variable dépendante chez le sexe k et $\varepsilon_{i j k}$ le paramètre d'effet aléatoire qui suit une distribution normale centrée de variance $\sigma_{\varepsilon}{ }^{2}$. Afin de tenir compte de notre contexte d'expérience naturelle, la valeur de référence pour déterminer 

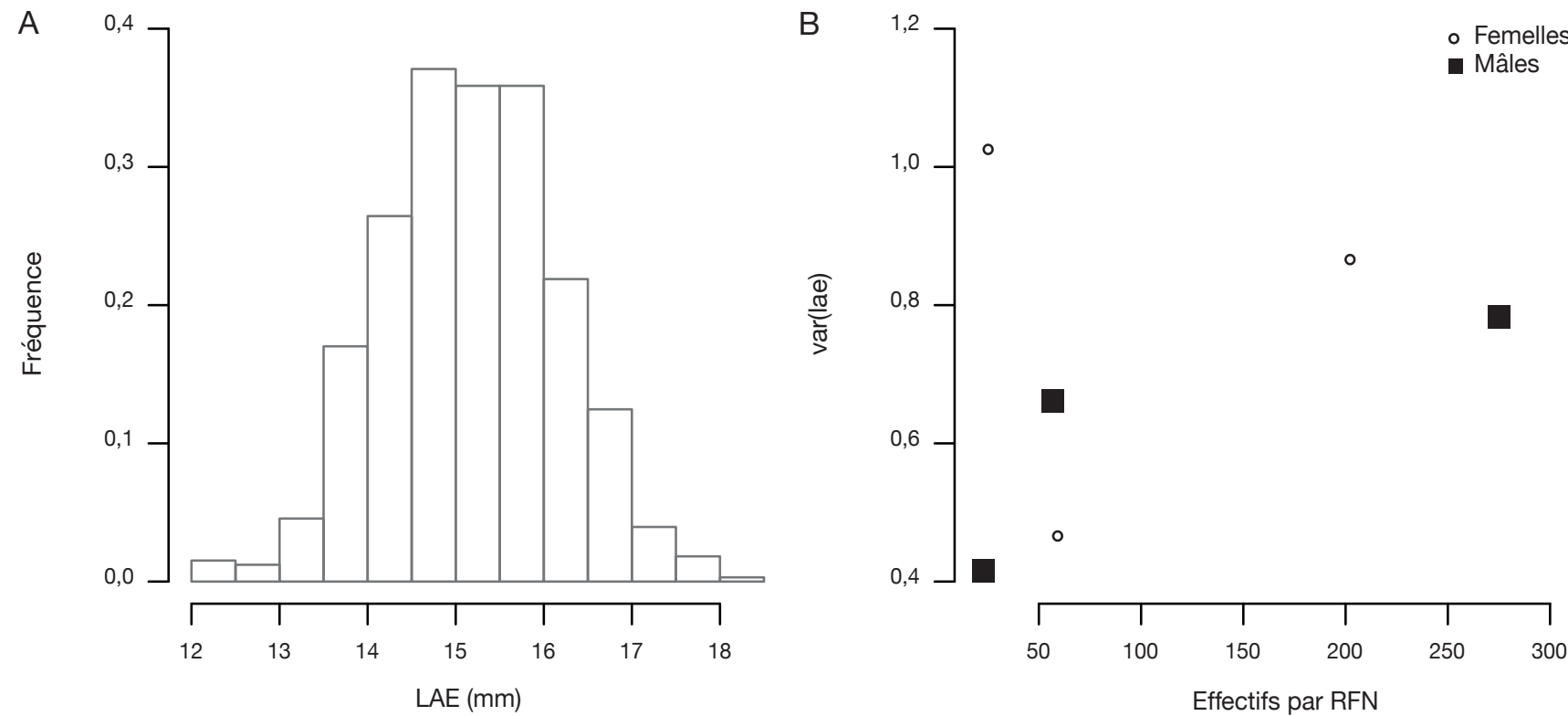

FIG. 5. - A, distribution observée de la longueur mesurée entre l'extrémité des mandibules et l'apex élytral (LAE) pour toutes les RFN de la Figure 3; B, variance de la longueur centrée réduite (lae) en fonction du sexe et du nombre de données disponibles dans les RFN 712 (Côte et arrière-côte de Bourgogne), 216 (Montagne Bourguignonne) et 544 (Plateau de Haye).

la probabilité, que l'hypothèse nulle engendre une statistique plus grande que celle calculée, est choisie pour correspondre à une probabilité de $5 \%$.

Le bootstrap stratifié a permis que les modèles soient toujours ajustés sur un nombre égal de mâles et de femelles par modalité du facteur étudié. Pour ajuster le modèle (1), on a généré n échantillons de 138 individus tirés au hasard avec remise, de manière à avoir toujours 23 mâles et 23 femelles par RFN. Le modèle est ajusté à chaque échantillon, de façon à produire $n$ valeurs des paramètres, dont la moyenne est l'estimateur bootstrap. Suivant la méthode préconisée par Davison \& Hinkley (1997), n a été fixé à 3000, après avoir testé des valeurs croissantes et surveillé la convergence de la variance des estimateurs. On a procédé de même pour l'ajustement des autres modèles.

Les données des stations représentées par plus de 10 individus ont été utilisées pour étudier le dimorphisme sexuel phénotypique en calculant les index comparatifs des moyennes (DSP) et écarts-types (ESP) des deux sexes. Pour plus de lisibilité et parce que nous n'en sommes pas encore au stade des comparaisons inter-spécifiques, nous n'avons pas utilisé la correction de mise à l'échelle de ESP que proposent Blanckenhorn et al. (2006). Dans cette étude d'une espèce, chez laquelle le dimorphisme sexuel est normalement à l'avantage des femelles, DSP s'écrit $\mathrm{DSP}_{\mathrm{k}}=1-\mu_{\mathrm{Fk}} / \mu_{\mathrm{Mk}}$. ESP se définit similairement comme $\mathrm{ESP}_{\mathrm{k}}=1-\sigma_{\mathrm{Fk}} / \sigma_{\mathrm{Mk}}$, avec (respectivement [resp.] mâles) $\mu_{\mathrm{Fk}} \mathrm{LAE}$ moyenne des femelles du site $k$ et $\sigma_{\mathrm{Fk}}$ la variance des femelles du site k. DSP (resp. ESP) est normalement négatif, s'annule dans le cas d'égalité des moyennes et devient positif dans celui de mâles ponctuellement plus gros que les femelles.

Les procédures statistiques ont été implémentées à l'aide de $\mathrm{R}$ version 3.3.2 ( $\mathrm{R}$ Core Team 2016) en utilisant le paquet boot (Canty $\&$ Ripley 2016). Les estimateurs des moyennes et des contrastes sont contraints à la précision de la mesure selon la méthode dite de "l'arrondi du banquier».

\section{RÉSULTATS}

Les valeurs d'aplatissement $(2,90)$ et de symétrie $(0,03)$ sont très faiblement éloignées de ce qui est attendu pour une distribution normale (Fig. 5A). Il n'y aura donc pas lieu de transformer la variable dépendante dans la suite de l'analyse. Notre individu le plus petit mesure $12,4 \mathrm{~mm}$ et le plus grand $18,3 \mathrm{~mm}$. La taille de nos spécimens a une espérance ( \pm écart-type) de 15,2 $\pm 1,0 \mathrm{~mm}$. Toutefois, une semblable normalité n'est pas le résultat escompté pour une espèce dimorphique. Cela laisse suspecter que le dimorphisme sexuel est masqué par d'autres sources de variation.

La variance de la variable dépendante centrée réduite a une évolution indépendante de l'effectif des échantillons de femelles, mais pas de celui des mâles. Chez ces derniers, elle augmente avec le nombre d'individus mesurés (Fig. 5B), ce que nous entendons contrôler par le biais du bootstrap stratifié.

Le modèle en fonction des RFN explique presque un quart de la variance totale (Tableau 1), en intégrant seulement deux facteurs. On retrouve le dimorphisme sexuel. Comme nous le constations pendant la phase de mesures, il se manifestait bel et bien entre mâles et femelles d'une même RFN qui sont plus petits en moyenne de $0,9 \mathrm{~mm} \pm 0,2\left(\mathrm{p}<7,58 \cdot 10^{-5}\right)$. On peut rejeter l'hypothèse nulle d'une variation fortuite de la taille imaginale entre les RFN au seuil de $\mathrm{p}<10^{-3}$. C'est bien cette variation interrégionale qui masquait le dimorphisme sexuel dans la Figure 5A.

Les deux sexes réagissent différemment dans le gradient latitudinal. Les populations femelles de Côte-d'Or ne different pas entre elles, si bien qu'on se retrouve avec une opposition nord-sud tranchée en faveur de la Côte-d'Or, où elles sont plus grosses de 0,7 mm sur la moyenne (Fig. 6) que dans la Haye. Dans les stations où les effectifs permettent le calcul des indices de dimorphisme, ESP (Fig. 7) les fait apparaître relativement plus variables que les mâles et laisse suspecter que leur variabilité stationnelle puisse annihiler ou masquer 


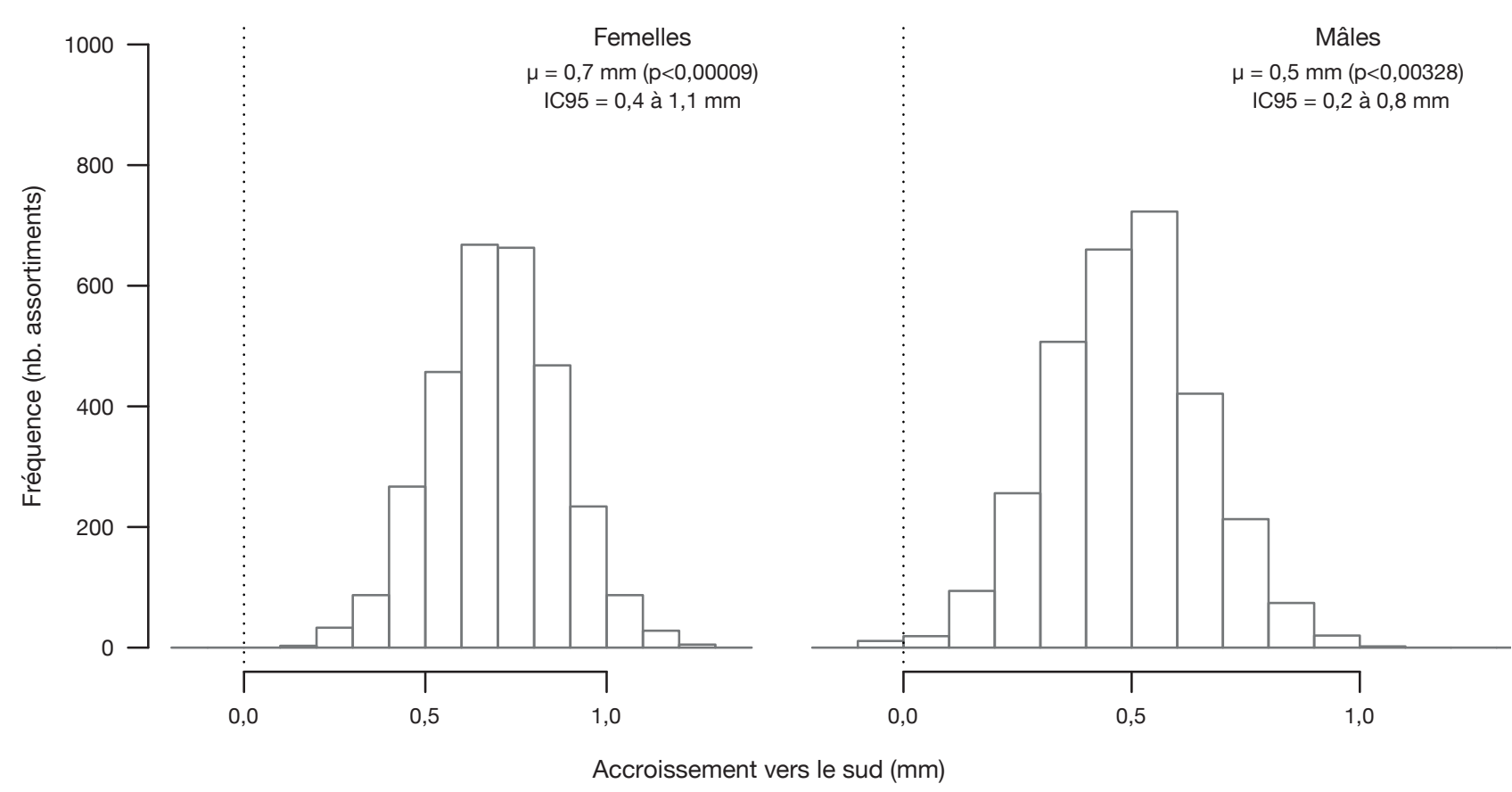

FIG. 6. - Accroissement de taille des femelles et des mâles en allant du nord au sud du transect. Contrastes calculés à partir de 3000 rééchantillonnages aléatoires avec remise à chaque extrémité du transect. La verticale pointillée demarque les tirages ayant généré un échantillon méridional plus grand (différence positive), de ceux où c'etait l'échantillon septentrional qui était le plus grand (différence negative). L'ordonnée indique le nombre d'assortiments dans chaque classe d'amplitude de différence. $\boldsymbol{\mu}$, la valeur moyenne de l'accroissement; IC95, l'intervalle de confiance à $95 \%$.

TABLEAU 1. - Partition de la variance calculée à partir des estimateurs bootstrap en fonction des facteurs Sexe, RFN, Altitude et Temps. Pour chaque partition sont indiquées en en-tête les RFN dont les données ont été utilisées pour l'ajustement (216: Montagne Bourguignonne; 544: Plateau de Haye; 712 : Côte et arriere-côte de Bourgogne). Abréviations: ${ }^{* \star}, \mathrm{p}<0,001 ;{ }^{* *}, \mathrm{p}<0,01 ;{ }^{*}, \mathrm{p}<0,05$; ddl, degrés de libertés; SC, somme des carrés; CM, carré moyen.

\begin{tabular}{|c|c|c|c|c|c|c|}
\hline Paramètre & ddl & SC & $\mathrm{CM}$ & $\mathbf{F}$ & $\operatorname{Pr}(>F)$ & \\
\hline \multicolumn{7}{|c|}{ Gradient latitudinal $(216,712$ \& 544) } \\
\hline Sexe & 1 & 109,20 & 109,16 & 142,48 & $<2,00 \cdot 10^{-16}$ & $\star \star \star *$ \\
\hline RFN/Sexe & 4 & 49,70 & 12,42 & 16,21 & $1,17.10^{-12}$ & $\star \star *$ \\
\hline Résidus & 638 & 488,80 & 0,77 & - & - & \\
\hline \multicolumn{7}{|c|}{ Gradient altitudinal (216 \& 712) } \\
\hline Sexe & 1 & 36,40 & 36,40 & 59,09 & $1,43 \cdot 10^{-12}$ & 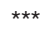 \\
\hline Altitude/Sexe & 4 & 5,89 & 1,47 & 2,39 & 0,0529 & \\
\hline \multicolumn{7}{|c|}{ Variation inter-générationnelle (216 \& 712) } \\
\hline Sexe & 1 & 41,61 & 41,61 & 55,84 & $2,21 \cdot 10^{-12}$ & $\star \star \star$ \\
\hline Époque/Sexe & 2 & 13,86 & 6,93 & 9,30 & $1,36.10^{-4}$ & $\star \star \star$ \\
\hline Résidus & 206 & 153,52 & 0,75 & - & - & \\
\hline
\end{tabular}

leur variabilité inter-stationnelle, jusqu'à éventuellement rendre indiscernables les femelles de RFN voisines. Il convient toutefois de considérer avec prudence cet aspect stationnel. À ce niveau d'observation, qui n'offre pas la même possibilité d'homogénéiser les effectifs mâles et femelles, qu'en agrégeant les données par RFN, on détecte une corrélation entre ESP et le sex ratio $\left(\mathrm{ESP}_{\mathrm{k}}=0,58 \cdot \rho_{\mathrm{k}}-0,95\right.$ avec $\mathrm{CME}_{\rho}=0,68$, $\mathrm{CME}_{\varepsilon}=0,10, \mathrm{p}<0,03815$, où $\rho_{\mathrm{k}}$ est le sex ratio pour le site $\mathrm{k}$ calculé comme le nombre de mâles pour le nombre de femelles, $\mathrm{CME}_{\rho}$ le carré moyen des écarts dûs au sex ratio et $\mathrm{CME}_{\varepsilon}$ le carré moyen résiduel), qui laisse augurer que ESP pourrait être influencé par les effectifs des deux sexes et donc par l'effectif de l'échantillon de la station. Pour le moment, on constate que le calcul d'un estimateur bootstrap stratifié par site et sexe, de manière à ne tester que des échantillons incorporant mâles et femelles en nombres égaux pour chaque site, ne conduit pas à une modification de la structure des données de la Figure 7.

Du côté des mâles, lae diffère significativement entre les extrémités nord et sud du transect (Fig. 6). Mais à l'inverse des femelles, ceux de la Montagne Bourguignonne sont plus gros (moyenne \pm écart-type) de $0,5 \pm 0,5 \mathrm{~mm}$, que leurs compères de la Côte $(\mathrm{p}<0,0323)$, soit d'une amplitude comparable au gradient nord-sud. Une variabilité intra-stationnelle moindre que celle des femelles pourrait conduire à des différences interstationnelles et générer une structure de leurs populations à un niveau inférieur à celui de la RFN. Mais en définitive, le dimorphisme sexuel demeure peu variable entre les stations et 


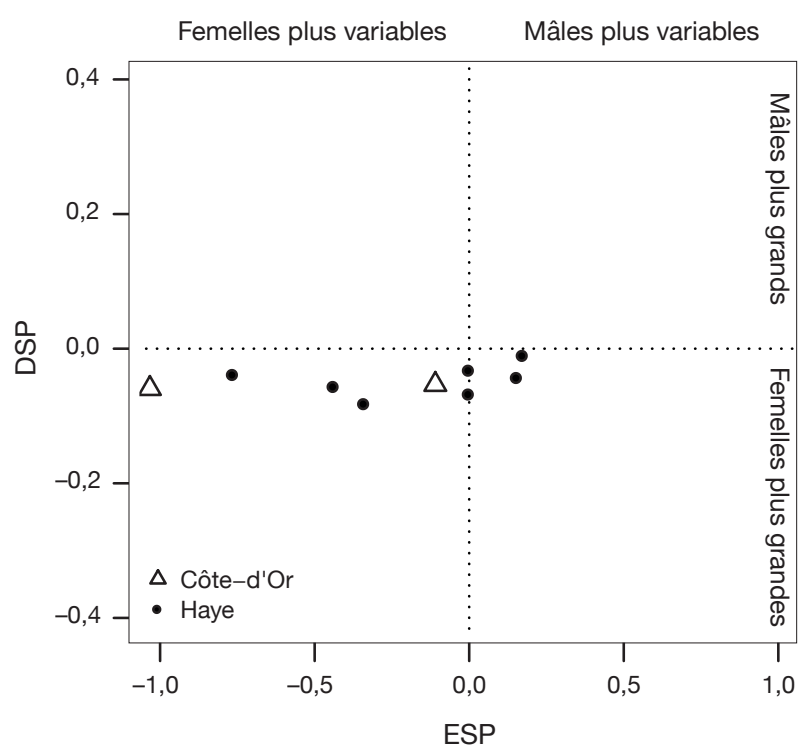

FIG. 7. - Variation du dimorphisme sexuel calculé pour les stations de Côte-d'Or et de Haye, representées par plus de 10 individus. Abréviations: DSP, rapport entre les tailles phénotypiques moyennes mâles et femelles; ESP, rapport entre les écart-types mâles et femelles.

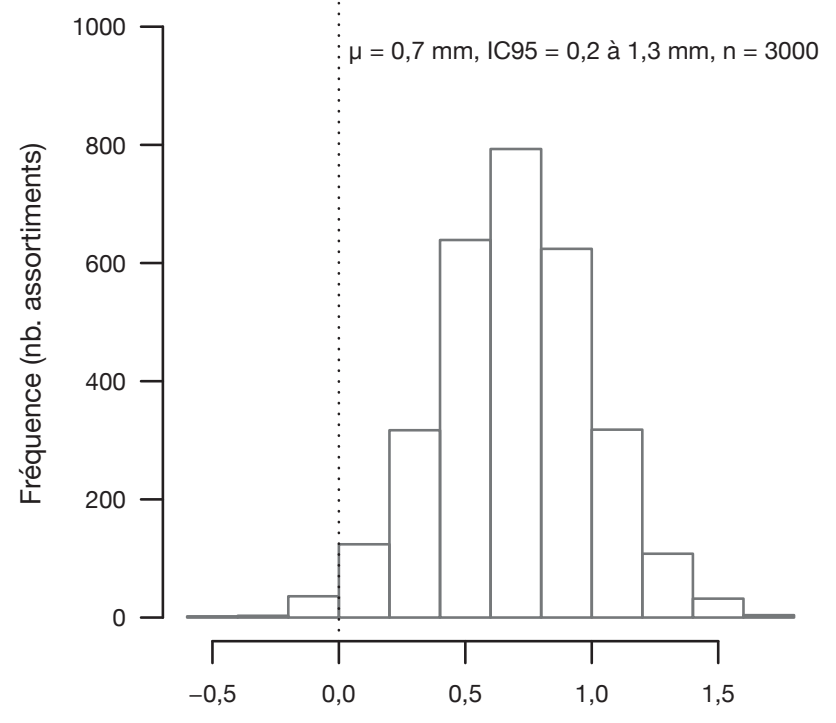

Différence femelles modernes-anciennes ( $\mathrm{mm})$

FIG. 8. - Distribution statistique de la différence de taille moyenne en millimètres entre 3000 assortiments de femelles de 1978-1998 (échantillons anciens) et 3000 assortiments de femelles de 2012-2016 (échantillons modernes). Chaque assortiment ancien (respectivement [= resp.] moderne) se compose de 14 femelles sélectionnées par un tirage aléatoire uniforme avec remise dans l'échantillon ancien (resp. moderne). Une valeur positive en abscisse signifie que l'assortiment moderne est en moyenne plus grand que l'assortiment ancien. La verticale pointillée démarque les tirages ayant généré un échantillon moderne plus grand, de ceux où c'etait l'échantillon ancien qui était le plus grand. L'ordonnée indique le nombre d'assortiments dans chaque classe d'amplitude de différence. $\boldsymbol{\mu}$, la différence moyenne de taille en millimètres entre les femelles modernes et anciennes, calculée à partir de la distribution; IC95, l'intervalle de confiance au seuil de $95 \%$.

n'indique pas de différence significative entre le nord et le sud (DSP dans la Figure 7), en sorte qu'il faut se ranger à l'idée que même si la structure latitudinale apparaît peu claire chez les mâles, ils finissent quand même par imiter les femelles.
TABLEAU 2. - Estimateurs bootstrap des contrastes en unités d'écart-type, calculés en ajustant le modèle 1 à 3000 échantillons aléatoires formés à partir des données 2012-2016 et 1978-1998 pour les RFN 216 (Montagne Bourguignonne) et 712 (côte et arrière-côte de Bourgogne). Mâles-femelles, différences de taille entre mâles et femelles; femelles XXI-XX, (respectivement [resp.] pour les mâles) différence de taille entre les femelles de l'échantillon 2012-2016 et celles de l'échantillon 1978-1998 (resp. pour les mâles); ${ }^{* * *}, p<0,001 ;{ }^{* *}, p<0,01 ;{ }^{*}, p<0,05$.

\begin{tabular}{lcccc}
\hline Paramètre & Moyenne & Écart-type & t & $\operatorname{Pr}(>|\mathbf{t}|)$ \\
\hline Intercept & 0,14 & 0,24 & $-0,60$ & - \\
Mâles-femelles & $-0,70$ & 0,33 & $-2,13$ & $0,0375^{*}$ \\
Femelles XXI-XX & $-0,73$ & 0,33 & 2,24 & $0,0290^{*}$ \\
Mâles XXI-XX & 0,44 & 0,31 & 1,39 & - \\
\hline
\end{tabular}

Quant à lui, le gradient altitudinal de Côte-d'Or réconcilie dans une indifférence égale les populations de la Montagne Bourguignonne et de la Côte (Tableau 1).

Quoique nous ne considérions pour l'instant qu'un pas de temps réduit, qui n'écarte que d'un peu plus d'une décennie la dernière observation du XXe siècle de la première du XXIe, cela semble suffisant pour capter une variation significative de la taille imaginale (gradient temporel dans le Tableau 1). Les mâles persistent à se singulariser par une réponse peu claire. Toute la variation est attribuable aux femelles. Celles d'avant 1998 ne sont pas tirées de la même distribution statistique, que celle sous-jacente aux données postérieures à 2012 (Tableau 2). En plus d'être significative, la différence peut être considérée comme importante au point de vue de sa magnitude, puisqu' elle exprime un écart entre les moyennes (Fig. 8). Avec 0,7 mm en faveur des femelles actuelles, on atteint la valeur de la variation trouvée le long du gradient latitudinal.

\section{DISCUSSION}

Le point de départ de cette analyse était notre besoin de confronter aux données notre pressentiment que la rédaction variétiste de Jeannel (1942) pointait en fait l'existence d'un cline latitudinal de taille phénotypique imaginale chez $P$. madidus. Par ailleurs, nous nous accordions avec Sukhodolskaya (2016) pour aussi déplorer un manque de connaissances sur ce sujet chez les coléoptères, qui n'étaient représentés que par quatre espèces dont un carabique dans les 58 insectes, desquels Chown \& Gaston (2010) synthétisèrent les résultats.

Un continuum se définit comme la production d'une variation sensible par une succession d'éléments adjacents indiscernables (Poincaré 1992). Dans le but d'arbitrer entre l'approche variétiste discrète de Jeannel (1942) et le point de vue clinal continu, nous avons discrétisé un transect nordsud de 300 kilomètres à l'aide des RFN, puis avons comparé les RFN les plus éloignées et celles contiguës. Étirer le transect et essayer de maximiser la variabilité édaphique en s'aventurant sur des terrains extrêmes aux yeux de $P$. madidus (Fig. 4) nous créa toutefois un embarras pour traiter le niveau stationnel, puisque cette stratégie nous menait à nous confronter à une importante disparité entre des sites avec peu de données et d'autres très populeux. 
TABLEAU 3. - Tous les cas trouvés chez $P$. madidus (Fabricius, 1775) de nanisme, celui-ci étant défini comme la manifestation d'une taille plus petite que ou égale à la limite inférieure de 13 millimètres, que la littérature prête à cette espèce au nord de l'Europe (cf. Tableau 1). Tous les sites sont localisés dans la RFN 544 (Plateau de haye). Station, identifiant du site; récolte, date de relevé du dispositif d'interception; étage, classe d'altitude, valant 2 pour les sites de 200 à 299 mètres et 3 pour les sites de 300 à 399 mètres; LAE, taille du spécimen.

\begin{tabular}{|c|c|c|c|c|c|c|}
\hline $\begin{array}{c}\text { Identifiant de } \\
\text { l'individu }\end{array}$ & Sexe & Station & Récolte & Étage & RFN & LAE (millimètres) \\
\hline \multicolumn{7}{|c|}{ Bois de Velaine-en-Haye (54) } \\
\hline 204 & femelle & VEL2 & 27/06/2008 & 3 & 544 & 12,5 \\
\hline 564 & mâle & VEL2B & 02/06/2009 & 3 & 544 & 13,0 \\
\hline 607 & mâle & VEL3B & $30 / 07 / 2008$ & 3 & 544 & 12,5 \\
\hline 609 & mâle & VEL3B & $22 / 05 / 2008$ & 3 & 544 & 12,4 \\
\hline 611 & mâle & VEL3B & $27 / 06 / 2008$ & 3 & 544 & 12,8 \\
\hline \multicolumn{7}{|c|}{ Bois de Gondreville (54) } \\
\hline 317 & mâle & GOND12 & $10 / 06 / 2008$ & 2 & 544 & 12,4 \\
\hline 358 & mâle & GOND6B & 02/06/2009 & 2 & 544 & 12,7 \\
\hline \multicolumn{7}{|c|}{ Bois de Maron (54) } \\
\hline 433 & mâle & MAR10B & 11/09/2008 & 3 & 544 & 13,0 \\
\hline 463 & mâle & MAR10B & $30 / 07 / 2008$ & 3 & 544 & 12,5 \\
\hline
\end{tabular}

C'est surtout pour les mâles que cette situation se révèle délicate. En effet, le dimorphisme sexuel de $P$. madidus ne se résume pas à la réalisation de tailles imaginales différentes, mais s'exprime aussi dans une structuration de la variance propre à chaque sexe. Les estimateurs fondés sur les données stationnelles en furent rendus imprécis. Ainsi, la corrélation identifiée entre la variance des mâles et l'effectif de l'échantillon contribue probablement aux variations de ESP. Les disparités d'effectifs entre les sites interdisaient de corriger le sex ratio, tout en homogénéisant le nombre de mâles entre les sites. C'est pourquoi une correction du sex ratio local ne modifiait pas la structure de la Figure 7. Pour ce qui concerne cet aspect, on doit se contenter à ce stade de se replier sur une position de prudence, en considérant que l'hypothèse nulle d'une variabilité localement identique des deux sexes ne peut pas être rejetée.

Chez les mâles se constate certes une différence significative entre les deux extrémités du transect, mais la structure clinale est brouillée par cette variabilité locale que nous peinons à capter, de sorte à ressortir sous une apparence de réaction molle confinant à l'inertie. Or, ce sont eux qui déterminent la borne inférieure de la plage de variabilité calculée par nos pairs, dont l'apparente stabilité pourrait avoir trouvé ici une explication. En prenant la latitude de la Haye comme point de repère, il vient deux remarques. La première concerne l'existence dès cette latitude de cas de nanisme (Tableau 3) « nordique", en ce sens qu'il n'est pas reporté de mâles plus petits que ceux-ci dans les Îles Britanniques (Lindroth 1974). La seconde est qu'en allant vers le sud de cette latitude, il y a une croissance de la taille imaginale mâle, puisque nous n'y rencontrons plus ces « nains » et que le dimorphisme sexuel paraît stable. En d'autres termes, il y a aussi à interroger la linéarité du cline mâle et l'existence d'un éventuel point latitudinal d'inflexion.

En agrément avec l'hypothèse de variation latitudinale continue, nous avons trouvé une différence sensible entre les femelles originaires de secteurs éloignés, cependant que nous faillissions à en détecter une entre les habitantes de RFN voisines. La discrétisation variétiste n'est donc pas un angle d'approche pertinent pour analyser la variabilité de $P$. madidus. Les femelles suivent un gradient de Bergmann inversé, cline connu pour émerger préférentiellement chez les espèces d'insectes univoltines de grande taille (Blanckenhorn \& Demont 2004). Les nôtres se révèlent plus grandes que leurs conspécifiques britanniques (Lindroth 1974) et plus petites que les géantes provençales (Jeannel 1942). Il y a eu de nombreux débats autour de la nature adaptative ou physiologique de ces phénomènes chez les poïkilothermes (voir Annexe 1), qui répondraient de la sorte au relâchement d'une contrainte. Il y aurait ainsi à tester l'hypothèse d'une relation entre le développement larvaire et la variation latitudinale de la durée de la belle saison (Blanckenhorn \& Demont 2004).

$P$. madidus paraît rejoindre le groupe déjà important des espèces connues pour enfreindre la règle de Rensch (Blanckenhorn et al. 2006) : le gradient latitudinal des mâles est affaibli et leur variabilité ne paraît pas significativement supérieure à celle des femelles. Comme beaucoup d'insectes, les carabiques démontrent également une corrélation entre fécondité et taille des femelles (Ernsting \& Huyer 1984). Une seconde hypothèse à tester serait donc celle d'une espèce, chez laquelle la pression de sélection pour la fécondité serait plus importante que celle pour la taille des mâles (Blanckenhorn et al. 2006).

Dans leur synthèse, le cline latitudinal le plus abrupt calculé à partir d'observations in natura par Blanckenhorn $\&$ Demont (2004) était de -3,8 \% pour l'orthoptère Teleogryllus yezoemma (Ohmachi \& Matsuura, 1951). Les mêmes indicateurs (Tableau 4) situent notre carabique à ce niveau. $P$. madidus se montre donc un des insectes les plus réactifs. En allant vers le nord, sa taille imaginale se réduit dans une proportion très supérieure aux $-0,8 \%$ trouvés à Carabus nemoralis O.F. Müller, 1764 en Allemagne (Blanckenhorn \& Demont 2004). Pour autant, il faut se garder de faire de P. madidus un cas particulier, car ces tests de gradient n'ont que peu impliqué les carabiques. Au contraire, on peut le rapprocher de Carabus granulatus Linnaeus, 1758, Carabus cancellatus Illiger, 1798 ou Pterostichus niger (Schaller, 1783). Chez les mâles russes ou biélorusses de ceux-ci s'observe une variation latitudinale complexe, parfois même cyclique, de la longueur des élytres, 
TABLEAU 4. - Variation de la taille imaginale moyenne en millimètres et pourcentages par degrés de latitude, selon que l'on prenne comme référence les points les plus extrêmes ou les plus rapprochés aux extrémités nord et sud du transect. Les latitudes nord et sud sont indiquées en degrés décimaux dans le système RGF93. La variation en pourcentage est calculée d'après la moyenne dans la Figure 6 comme $\hat{\delta}_{\text {LAE }} /\left(\hat{\mu}(\mathrm{s})\right.$ j. $\hat{\delta}_{\text {Lat }}$ ) où $\hat{\delta}_{\text {LAE }}$ (mm) est l'estimateur de la variation entre les extrémités du transect (ligne du dessus), $\hat{\mu}(\mathrm{s})_{j}$ est l'estimateur de la LAE moyenne à l'extrémité sud pour le sexe considéré et $\hat{\delta}_{\text {Lat }}$ est l'extension latitudinale du transect.

\begin{tabular}{|c|c|c|}
\hline Valeur & Gradient le plus long & Gradient le plus court \\
\hline Station sud & RNCLJR70/1 & CC9 \\
\hline Latitude sud $\left({ }^{\circ}\right)$ & 47,21826 & 47,61450 \\
\hline Station nord & GOND6B & TAG6 \\
\hline Latitude nord $\left(^{\circ}\right)$ & 48,68166 & 48,56204 \\
\hline Gradient & 1,46340 & 0,94754 \\
\hline Variation moyenne des femelles en $\mathrm{mm}$ (et en \% par degrés de latitude) & $-0,7(-3,08)$ & $-0,7(-4,75)$ \\
\hline Variation moyenne des mâles en $\mathrm{mm}$ (et en \% par degrés de latitude) & $-0,3(-2,04)$ & $-0,5(-3,40)$ \\
\hline
\end{tabular}

cependant que les femelles expriment là-bas aussi un cline net (Sukhodolskaya 2016). La longueur des élytres de C. granulatus décroît de $5 \mathrm{~mm}$ en se déplaçant de 4,07 degrés de latitude vers le nord (Sukhodolskaya 2016; fig. 4). Cela fait une variation de $-9,5 \%$ par degré depuis le sud, soit une réduction de taille très supérieure à celle que nous observons à chacun des deux sexes de $P$. madidus.

La difficulté à se procurer des spécimens anciens correspondant à nos critères nous a conduits à considérer un échantillon historique d'une taille assez réduite, mais finalement pas autant que dans le cas de certains des échantillons de Sukhodolskaya (2016). Pour la suite, nous escomptons lui ajouter d'anciens relevés effectués dans d'autres RFN de Côte-d'Or, en face desquelles il suffira de mettre les résultats de nouvelles collectes. Pour le moment, l'échantillon ancien se compose déjà de spécimens obtenus de plusieurs localités distantes, ce qui nous met au moins à l'abri d'un éventuel artefact stationnel.

Le test historique avait originellement été envisagé en tant qu'une étape de contrôle, afin de vérifier la faisabilité de comparer des relevés asynchrones, notre hypothèse a priori était une identité des spécimens passés et contemporains. Elle s'est trouvée déjouée pour les femelles. Ayant reproduit le protocole de naturalisation du muséum pour vérifier qu'il n'en résultait pas de différences pour les seules femelles, le résultat de ce test peut se voir sous deux angles différents. Dans l'immédiat, il nous dit que la désynchronisation entre les extrémités du transect, quoiqu'elle représentât un intervalle de temps bien moindre, conduit quand même à ce que nos résultats latitudinaux incorporent, dans une proportion inconnue, un peu cette tendance à l'accroissement de la taille imaginale au fil du temps. À quelques exceptions près, la dimension temporelle est généralement méconnue par les études de gradients géographiques, comme Sukhodolskaya (2016) dont les échantillons couvraient le même intervalle de temps que notre test historique. Comme nous-mêmes l'indiquions plus haut, de précédents résultats avaient suggéré une incidence négligeable de la variabilité inter-annuelle. Mais il ne s'était agi que de comparer des espèces de coléoptères sur quatre années consécutives (Gouws 2007). De notre côté, nous ignorons si ce que nous venons de mettre en évidence chez $P$. madidus peut se généraliser à d'autres espèces de carabiques. Mais nous ne pouvons non plus exclure que d'autres résultats de clines latitudinaux que les nôtres puissent avoir été pareillement parasités.
Plusieurs cas de changements inter-générationnels de la taille imaginale ont été documentés chez les Arthropodes. Parfois, ce sont des fluctuations annuelles (Claus-Walker et al. 1997). D'autres sont soutenus dans le temps, plus particulièrement chez les femelles (Gilchrist et al. 2004). Ils apparurent dans des situations telles que:

- le transfert des spécimens du milieu naturel vers le laboratoire (David et al. 1997);

- l'introduction de l'espèce hors de son aire naturelle (Huey et al. 2000);

- une variation de l'intensité de prédation sur les larves (Claus-Walker et al. 1997; Manyak 2012);

- une fluctuation de l'accès aux ressources (Gouws 2007).

Pour l'ordre des Coléoptères, un cas de réponse au changement climatique a été identifié chez un Gyrinidae américain (Babin-Fenske et al. 2008). L'observation faite ici apporte deux faits originaux, puisqu'il s'agit d'un premier cas de coléoptères terrestre et qu'il est observé dans son milieu naturel.

Quoique nous ayons peu à reprendre de Babin-Fenske et al. (2008), puisque les animaux aquatiques sont amenés à réagir aux variations de paramètres physico-chimiques propres à leur habitat (Horne et al. 2015), il reste de ces exemples qu'un changement inter-générationnel de la taille imaginale constitua à chaque fois une réponse de l'animal à une modification d'un ou de plusieurs paramètres édaphiques (voir Annexe 1) ou biotiques de l'environnement de développement larvaire. En rapportant cette constatation à notre sujet, nous sommes amenés à inférer de nos données qu'un changement environnemental perceptible par $P$. madidus s'opérerait depuis au moins 14 ans, à l'échelle spatiale d'un département.

Il y a peu de paramètres relatifs à l'écophysiologie larvaire qui nous soient accessibles pour le moment. Pour ce qui concerne le volet gestion, on peut écarter l'hypothèse qu'un changement d'usage des sols ait affecté la chaîne trophique, car il n'y en a pas eu dans ces forêts publiques. Le principal événement historique fut durant la dernière décennie la constitution de réserves, qui s'interdisent les travaux sylvicoles sur tout ou partie de leur territoire, précisément dans l'idée qu'il en résulterait une amélioration de l'habitat pour les faunes forestières. Notre volonté initiale d'exploration des structures spatiales découlait en partie d'un désir de tester l'existence d'un « effet réserve ». C'est pourquoi l'échantillonnage a aussi couvert des boisements côte-d'oriens dévolus à la production, 
choisis isolés ou à une distance assez importante pour minimiser le risque d'échanges pour les espèces marcheuses, dont quelques-uns en forêt privée. À ce stade, leurs données ne se sont individualisées dans les tendances résiduelles, ni à l'occasion de l'étude de gradient latitudinal, ni dans le test historique. Une étude à une échelle spatiale plus fine, trouvée nécessaire pour comprendre la structuration de la variance des mâles, apportera peut-être aussi des précisions quant aux femelles.

Sur la base de l'intervalle de confiance à $95 \%$ (Fig. 8), les femelles auraient pris en moyenne entre 14 et 93 microns à chaque génération. Au moment de nos expériences, la durée d'activité saisonnière de $P$. madidus s'appréciait de 1,21 jours par an en moyenne en Grande-Bretagne, ce que Pozsgai \& Littlewood (2011) imputèrent au changement climatique, sachant qu'une relation embryologique avec les paramètres climatiques a déjà été trouvée à cette espèce (Terrell-Nield 1990).

Des méta-analyses ont été publiées pour alerter sur la forte probabilité d'une variation de la taille des ectothermes en réponse à une élévation de température. Des éléments expérimentaux commencent à les corroborer. Par exemple, le crapaud commun Bufo bufo (Linnaeus, 1758) démontra en Grande-Bretagne le cas opposé au nôtre d'un rétrécissement, qui s'avéra encore une fois le plus sensible chez les femelles, dont l'hibernation est dérangée par des températures trop clémentes (Reading 2007).

La démonstration formelle d'un lien entre la variation historique de la taille de $P$. madidus et le changement climatique reste à produire, car elle n'était pas l'objectif de ce travail-ci, qui n'en apporte que les indices, ainsi que le firent avant nous Pozsgai \& Littlewood (2011). La qualité et la quantité des ressources trophiques disponibles pour la larve (Luff 1974) nous sont inconnues. Mais il ressort qu'il vaut la peine de ranger $P$. madidus parmi les espèces candidates pour valider expérimentalement le modèle de Horne et al. (2015), d'après lequel il y a une forte probabilité que la réaction d'une espèce arthropode à une élévation de température puisse se déduire de son cline latitudinal. Nous viennent aussi des questionnements sur les conséquences écologiques de tels changements, comme: - si cette croissance des femelles se confirmait, a-t-elle une limite supérieure et quand aurait-elle commencé ?

- cette croissance des femelles pourrait-elle résulter en une amélioration de la fécondité et ainsi modifier la dynamique des populations?

- ces résultats sont-ils transposables à d'autres espèces à reproduction automnale et y a-t-il à attendre d'un allongement de la saison de végétation, qu'il se traduise par un changement de l'équilibre des faunes carabidologiques en faveur des espèces à reproduction automnale?

Pour simples qu'elles puissent trompeusement apparaître, ces interrogations recouvrent en réalité de nombreux et complexes aspects de l'histoire naturelle des différentes espèces, qu'il n'est pas encore temps ici de développer. À ce stade, l'important est que ces questions se décomposent en hypothèses qui peuvent faire l'objet de tests expérimentaux, qui constituent la prochaine étape nécessaire de cette recherche.

\section{CONCLUSION}

Nous avons pu établir les caractéristiques de la distribution statistique de la taille imaginale de $P$. madidus. Mais ce travail n'est pas achevé. Nous avons pu mettre en évidence un gradient latitudinal qui affecte au moins les femelles, mais il y a matière à suspecter une structure localement plus complexe des populations de mâles. L'existence des spécimens nains a été confirmée, mais nous n'avons pas observé les géantes, qui doivent vivre bien plus au sud.

Il nous a paru nécessaire d'en passer par cette phase descriptive préliminaire, car nous nous penchions sur un domaine de la coléoptérologie, qui faisait l'objet d'un relatif désinvestissement en comparaison de ce qui se fait sur les autres ordres d'insectes. Nous sommes donc restés autant que possible dans le cadre d'une étude de corrélations. Une explication du phénomène reste à construire. On peut raisonnablement anticiper que cette affaire ne sera pas moins ardue que pour les autres poïkilothermes, mais l'effort se justifie par son actualité.

Nous avons ajusté un modèle d'une simplicité scolaire à une variable dépendante facile à mesurer, relevée sur une espèce banale et nous nous apercevons que cela soulève beaucoup de questions. Les espèces banales sont trop souvent délaissées. Avec ce cas, on voit qu'il y a pourtant encore à apprendre sur leur compte. C'est même sûrement pour celles-ci que se peuvent nourrir les meilleurs espoirs d'une progression des connaissances éco-entomologiques, précisément parce que leur abondance favorise le recours aux méthodes de l'écologie quantitative, tout en maintenant l'effort expérimental dans des limites raisonnables. Dans le cas de P. madidus, nous sommes confiants dans la possibilité de progresser, car il n'y a justement pas à craindre de venir à manquer de spécimens.

\section{Remerciements}

Nous tenons à exprimer notre gratitude aux relecteurs dont Arnaud Horellou pour leurs remarques qui ont fait avancer le traitement de ce sujet, à Stéphane Puissant (MJSD) qui a aussi participé aux relectures, ainsi qu'aux initiateurs de ces travaux, dont certains sont partis depuis exercer leurs talents sous d'autres latitudes: Jean-Luc Dupouey (INRA Nancy), Laurent Godé (PNR Lorraine), Rogier Leysen (ONF), Laurent Servière (RNF), Thomas Morant (LPO21), Stéphane Woynaroski (CR Bourgogne-Franche-Comté), la DREAL Bourgogne-Franche-Comté, la Communauté de communes Nuits-Saint-Georges-Gevrey-Chambertin et la Communauté de communes Pays de Seine et Tille.

\section{RÉFÉRENCES}

Babin-Fenske J., ANAND M. \& Alarie Y. 2008. — Rapid morphological change in stream beetle museum specimens correlates with climate change. Ecological Entomology 33 (5): 646-651. https:// doi.org/10.1111/j.1365-2311.2008.01018.x

BlancKenhorn W. U. \& Demont M. 2004. - Bergmann and 
converse Bergmann latitudinal clines in arthropods: Two ends of a continuum? Integrative and Comparative Biology 44: 413-424. https://doi.org/10.1093/icb/44.6.413

BlanCKenHorn W. U., STILlWell R. C., Young K. A., Fox C. W. \& Ashton K. G. 2006. - When Rensch meets Bergmann: does sexual size dimorphism change systematically with latitude? Evolution 60: 2004-2011. https://doi.org/10.1554/06-110.1

BUTTERFIELD J. 1996. — Carabid life-cycle strategies and climate change: a study on an altitude transect. Ecological Entomology 21: 9-16. https://doi.org/10.1111/j.1365-2311.1996.tb00260.x

CANTY A. \& RiPLEY B. 2016. - Bootstrap Functions (Originally by Angelo Canty for S). R Foundation for Statistical Computing, Vienna, Austria, $117 \mathrm{p}$

CAVAIGNAC S. 2009. - Les sylvoecoregions (SER) de France métropolitaine, étude de définition. Rapport, Inventaire forestier national, Nogent-sur-Vernisson, 166 p.

ChOwN S. L. \& GASTON K. J. 2010. - Body size variation in insects: a macroecological perspective. Biological Reviews 85: 139-169. https://doi.org/10.1111/j.1469-185x.2009.00097.x

Claus-Walker D. B., Crowley P. H. \& Johansson F. 1997. — Fish predation, cannibalism, and larval development in the dragonfly Epitheca cynosura. Canadian Journal of Zoology 75: 687-696. https://doi.org/10.1139/z97-089

Coulon J., Pupier R., Queinnec E., Ollivier E. \& Richoux P. 2011. - Coléoptères carabiques. Compléments et mises à jour. Volume 2. Faune de France. Féderation francaise des societes de sciences naturelles, $337 \mathrm{p}$.

David J. R., Gibert P., Gravot E., Petavy G., Morin J.-P., Karan D. \& Moreteau B. 1997. - Phenotypic plasticity and developmental temperature in Drosophila: analysis and signicance of reaction norms of morphometrical traits. Journal of Thermal Biology 22: 441-451. https://doi.org/10.1016/S03064565(97)00063-6

DAVISON A. C. \& HiNKLEY D. V. 1997. — Bootstrap methods and their application. Volume 1. Cambridge university press, Cambridge, $582 \mathrm{p}$

ERNSTING G. \& HuYER F. 1984. - A laboratory study on temperature relations of egg production and development in two related species of carabid beetles. Oecologia 62: 361-367. https://doi.org/10.1007/BF00384268

ERNSTING G. \& ISAAKS J. 1987. — Effects of food intake and temperature on energy budget parameters in Notiophilus biguttatus (Coleoptera Carabidae). Acta Phytopathologica et Entomologica Hungarica 22: 135-145.

ERNSTING G. \& ISAAKS J. 1997. — Effects of temperature and season on egg size, hatchling size and adult size in Notiophilus biguttatus. Ecological Entomology 22: 32-40. https://doi.org/10.1046/j.13652311.1997.00040.x

Ernsting G., IsaAKs J. \& Berg M. 1992. - Life cycle and food availability indices in Notiophilus biguttatus (Coleoptera, Carabidae). Ecological Entomology 17: 33-42. https://doi. org/10.1111/j.1365-2311.1992.tb01036.x

Gilchrist G. W., Huey R. B., Balanya J., Pascual M. \& Serra L. 2004. - A time series of evolution in action: a latitudinal cline in wing size in south american Drosophila subobscura. Evolution 58: 768-780. https://doi.org/10.1554/03-414

Gouws E. J. 2007. - Intraspecic body size variation in insects. Thèse, Stellenbosch University, Stellenbosch, 117 p.

Horne C. R., Hirst A. G. \& ATKINSON D. 2015. - Temperaturesize responses match latitudinal-size clines in arthropods, revealing critical differences between aquatic and terrestrial species. Ecology Letters 18: 327-335. https://doi.org/10.1111/ele.12413

Huey R. B., Gilchrist G. W., Carlson M. L., Berrigan D. \& SERRA L. 2000. - Rapid evolution of a geographic cline in size in an introduced fly. Science 287: 308-309. https://doi.org/10.1126/ science. 287.5451 .308

INVENTAIRE FORESTIER NATIONAL 2008. - Inventaire Forestier Départemental. Côte-d'Or. 4e inventaire 2004. Rapport. Ministère de l'Agriculture et de la Pêche, Nogent-sur-Vernisson, 168 p.

JEANNEL R. 1942. - Coléoptères carabiques: II. Faune de France. Lechevalier, Paris, 40: 572-1173.

Kotze D. J., Brandmayr P., Casale A., Dauffy-Richard E., DeKOninCK W., KOIVUla M. J., LÖVEI G. L., MOSSAKOWSKI D., Noordijk J., Paarmann W., Pizzolotto R., Saska P., Schwerk A., Serrano J., Szyszko J., Taboada A., Turin H., Venn S., Vermeulen R. \& ZeTto T. 2011. - Forty years of carabid beetle research in europe-from taxonomy, biology, ecology and population studies to bioindication, habitat assessment and conservation. ZooKeys 100: 55-148. https://doi.org/10.3897/ zookeys. 100.1523

LiNDROTH C. H. 1974. - Handbooks for the identication of british insects. Coleoptera, Carabidae. Royal Entomological Society of London, Londres, $155 \mathrm{p}$.

LuFf M. L. 1974. - Adult and larval feeding habits of Pterostichus madidus (F.)(Coleoptera: Carabidae). Journal of Natural History 8:403-409.

MANYAK A. 2012. - A latitudinal body size pattern in a marine isopod suggests local adaptation to predator risk. Thèse, College of Charleston, Charleston, $59 \mathrm{p}$.

POINCARÉ H. 1992. - La science et l'hypothèse. Éditions de la Bohème, Rueil-Malmaison, $310 \mathrm{p}$.

POZSGAI G. \& LitTLEWOOD N. A. 2011. — Changes in the phenology of the ground beetle Pterostichus madidus (Fabricius, 1775) Insect science 18: 462-472. https://doi.org/10.1111/j.17447917.2011.01416.x

R CORE TEAM 2016. - R: A Language and Environment for Statistical Computing. R Foundation for Statistical Computing, Vienna, Austria. https://www.R-project.org/ (dernière consultation le 21 mai 2018)

READING C. 2007. - Linking global warming to amphibian declines through its effects on female body condition and survivorship. Oecologia 151: 125-131. https://doi.org/10.1007/ s00442-006-0558-1

SCHREINER A. \& SCHWERK A. 2012. - Does the mean individual biomass (MIB) of carabids as a bioindicator of forest succession follow a logistic function? examples from western german beech and polish scots pine forests. Baltic Journal of Coleopterology 12: 7-64.

Soares A. O., Coderre D. \& Schanderl H. 2003. - Effect of temperature and intraspecic allometry on predation by two phenotypes of Harmonia axyridis Pallas (Coleoptera: Coccinellidae). Environmental Entomology 32: 939-944. https://doi. org/10.1603/0046-225X-32.5.939

SUKHODOLSKAYA R. 2016. - Intra-specic body size variation of ground beetles (Coleoptera: Carabidae) in latitudinal gradient. Periodicum Biologorum 118: 273-280. https://doi.org/10.18054/ pb.2016.118.3.3918

TERRELL-Nield C. 1990. - Distribution of leg-colour morphs of Pterostichus madidus (F.) in relation to climate, in N. STORK (éd.), The role of ground beetles in ecological and environmental studies. Intercept, Andover, 39-51.

ToÏgo M., Paillet Y., Noblecourt T., SOldati F., Gosselin F. \& DAUFFY-RICHARD E. 2013. — Does forest management abandonment matter more than habitat characteristics for ground beetles? Biological Conservation 157: 215-224. https://doi.org/10.1016/j. biocon.2012.07.025

TyLER G. 2008. - Differences in abundance, species richness, and body size of ground beetles (Coleoptera: Carabidae) between beech (Fagus sylvatica L.) forests on podzol and cambisol. Forest Ecology and Management 256: 2154-2159. https://doi.org/10.1016/j. foreco.2008.08.006 


\section{ANNEXE}

ANNEXE 1. - Glossaire.

Allométrique: dans ce travail ce terme est employé dans le sens de l'allométrie statique, qui désigne la covariation interindividuelle non proportionnelle des caractères.

Calcaires bajociens : terme de la nomenclature stratigraphique géologique qui désigne un étage essentiellement calcaire, mais aussi marneux et argileux produit au Jurassique moyen (-170,3 $\pm 1,4$ à $-168,3 \pm 1,3$ millions d'années) dont le stratotype a été défini en 1859, par A. d'Orbigny et qui correspond en Côte-d'Or à l'épisode tectonique du rifting thétysien.

Calcaires bathoniens: terme de la nomenclature stratigraphique géologique qui désigne un étage calcaire du Jurassique moyen $(-168,3 \pm 1,3$ à $-166,1 \pm 1,2)$ défini par d'Halloy en 1843, nommé d'après la ville de Bath (RU), séparé du bajocien en Côte-d'Or par une couche plus détritique témoignant de l'ennoyage généralisé de la plateforme bajocienne par la Thétys, sous laquelle elle a formé un haut fond au large de l'île du Massif Central, entre la Laurasie et le Gondwana.
Cuesta: relief produit par l'érosion d'une couche sédimentaire tendre inclinée, qui surplombe une couche résistante. Là où ne reste que la couche résistante se forme le front vertical, en arrière duquel descend un plateau incliné.

Édaphiques : adjectif dérivé de édaphisme, qui est l'influence des caractères physico-chimiques d'un milieu sur les organismes qui y vivent.

Graticule : réglette de référence utilisée en microscopie pour calculer la longueur réelle représentée par les graduations de l'oculaire du microscope et éventuellement mesurer les déformations optiques à la périphérie du champ.

Poïkilotherme: organisme à température interne variable. Il existe deux catégories de poïkilothermes. Les poïkilothermes endothermes ont une production variable de chaleur corporelle (exemple: mammiferes capables d'hibernation). Les poïkilothermes ectothermes, groupe qui inclue les insectes, n’ont pas de régulation interne de leur température corporelle. Substratum : couche géologique qui supporte le sol. 